\title{
Estimates of Mean-Type Fractional Inequalities For Differentiable Functions
}

Muhammad Samraiz ${ }^{1}$, Zahida Perveen ${ }^{2}$, Sajid Iqbal ${ }^{3}$, Saima Naheed ${ }^{2}$, and Thabet Abdeljawad $^{4}$

${ }^{1}$ University of Sargodha

${ }^{2}$ University of Sargodha Faculty of Sciences

${ }^{3}$ Riphah International University - Lahore Campus

${ }^{4}$ Prince Sultan University

June 12,2021

\begin{abstract}
In this article, we established a wide range of fractional mean-type integral inequalities for notable Hilfer fractional derivative using twice differentiable convex and $\$ \mathbf{s} \$$-convex functions for $\$ \mathbf{s} \backslash \operatorname{in}(0,1] \$$ with related identities. Also the results for Caputo fractional derivatives are derived as a special case of our general results.
\end{abstract}

\section{Hosted file}

M.Samraiz, Z. Perveen ,S. Iqbal, Saima Naheed, Thabet Abdeljawad.pdf available ractional-

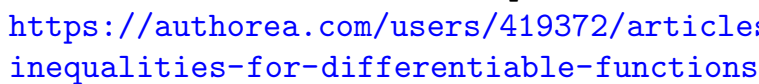

